\title{
Simulating an invasion: unsealed water storage (rainwater tanks) and urban block design facilitate the spread of the dengue fever mosquito, Aedes aegypti, in Brisbane, Australia
}

\author{
Brendan J. Trewin $(1) \cdot$ Hazel R. Parry • Daniel E. Pagendam • Gregor J. Devine • \\ Myron P. Zalucki · Jonathan M. Darbro · Cassie C. Jansen • Nancy A. Schellhorn
}

Received: 13 November 2020/Accepted: 13 August 2021 / Published online: 25 August 2021

(C) The Author(s), under exclusive licence to Springer Nature Switzerland AG 2021

\begin{abstract}
Aedes aegypti (Linnaeus) was once highly prevalent across eastern Australia, resulting in epidemics of dengue fever. Drought conditions have led to a rapid rise in semi-permanent, urban water storage containers called rainwater tanks known to be critical larval habitat for the species. The presence of these larval habitats has increased the risk of establishment of highly urbanised, invasive mosquito vectors such as Ae. aegypti. Here we use a spatially explicit network model to examine the role that unsealed rainwater tanks may play in population connectivity of an Ae. aegypti invasion in suburbs of Brisbane, a major Australian city. We characterise movement between rainwater tanks as a diffusion-like process, limited by
\end{abstract}

Supplementary Information The online version contains supplementary material available at https://doi.org/10.1007/ s10530-021-02619-z.

B. J. Trewin $(\bowtie) \cdot$ H. R. Parry · D. E. Pagendam •

N. A. Schellhorn

Commonwealth Scientific and Industrial Research

Organisation, Health and BiosecurityBusiness Unit,

Brisbane, Australia

e-mail: brendan.trewin@csiro.au

B. J. Trewin - G. J. Devine · J. M. Darbro

Mosquito Control Laboratory, QIMR Berghofer Medical

Research Institute, Brisbane, Australia

B. J. Trewin - M. P. Zalucki

School of Biological Sciences, The University of

Queensland, St Lucia, Brisbane, Australia a maximum distance of movement, average life expectancy, and a probability that Ae. aegypti will cross wide open spaces such as roads. The simulation model was run against a number of scenarios that examined population spread through the rainwater tank network based on non-compliance rates of tanks (unsealed or sealed) and road grids. We show that Ae. aegypti tank infestation and population spread was greatest in areas of high tank density and road lengths were shortest e.g. cul-de-sacs. Rainwater tank noncompliance rates of over $30 \%$ show increased connectivity when compared to less than $10 \%$, suggesting rainwater tanks non-compliance should be maintained under this level to minimize the spread of an invading Ae. aegypti population. These results presented as risk maps of Ae. aegypti spread across Brisbane, can assist health and government authorities on where to

\author{
J. M. Darbro - C. C. Jansen \\ Metro North Public Health Unit, Queensland Health, \\ Windsor, Brisbane, Australia \\ C. C. Jansen \\ Communicable Diseases Branch, Department of Health, \\ Queensland Health, Herston, Australia
}


optimally target rainwater tank surveillance and educational activities.

Keywords Aedes aegypti · Network model · Invasion simulation · Population spread · Water storage

\section{Introduction}

Large epidemics of dengue historically plagued the east coast of Australia, where an estimated 70-90\% of the human population were infected (Lumley and Taylor 1943). It has been suggested that large water storage containers, called rainwater tanks, maintained Aedes aegypti (Linnaeus) populations responsible for these epidemics (Hamlyn-Harris 1931; Lumley and Taylor 1943). The removal of rainwater tanks from major urban environments is thought to have contributed to the disappearance of the species from subtropical locations (Russell et al. 2009; Trewin et al. 2017). However, drought conditions in the early 2000 s and subsequent government incentives, saw a rapid rise in the number of rainwater tanks reinstalled throughout all major Australian cities. It is estimated that there are now $\sim 300,000$ rainwater tanks in the south-east Queensland region, representing nearly $40 \%$ of residential dwellings (Moglia et al. 2013). When unsealed or 'non-compliant' with regulations, rainwater tanks are ideal habitat for all life-cycle stages of Ae. aegypti and have the potential to act as a major population source for disease vectors (Trewin et al. 2020; Tun-Lin et al. 1995). Rainwater tanks provide all the requirements for development of the species; reliable larval food resources, ideal temperature range and humidity, a surface for oviposition and resting, a permanent or semi-permanent source of water where larval density is unlikely to be restricted and close proximity to human hosts (Trewin 2018). The rapid rise of these permanent water storage containers has led to an increased risk of the reestablishment of Ae. aegypti in Brisbane, the largest urban centre in Queensland, Australia (Heersink et al. 2015; Trewin 2018; Trewin et al. 2013).

Population connectivity is an important ecological process that underpins the ability of an organism to persist and spread within a landscape (Fahrig and Merriam 1985). Connectivity is best defined as the capacity of a landscape to facilitate or impede the movement of organisms between resource patches (Taylor et al. 1993). Therefore, persistence and spread of a newly established organism can be influenced by movement patterns and mortality as a function of landscape structure (Ferrari et al. 2007). In medically important species such Ae. aegypti, an understanding of landscape connectivity guides vector management practices (State of Queensland 2015) or optimizes 'rear and release' strategies such as population replacement (Hoffmann et al. 2011) or the sterile insect technique (Dyck et al. 2006). For instance, the removal of larval sources, a tenet of mosquito control for decades, aims to suppress or eliminate a population by removing key habitat resources within a landscape (Gorgas 1915; Gubler and Clark 1994; Trewin et al. 2017). In this way connectivity between resource patches is disrupted, lowering population spread and abundance, the probability of persistence and the prevention of disease transmission. A better understanding of Ae. aegypti population spread, limited by the spatial distribution of resource abundance, could lead to improved control programs and strategies for achieving elimination.

As computer power and digital tools have improved, so have opportunities to explore complex biological processes such as population spread with modelling approaches. Spatially explicit models are a method by which the connectivity of mosquito populations can be explored without the need for expensive field experiments and data collection. In models such as these, population dynamics and movement are simulated across representative landscapes in order to identify movement pathways between key resources (Ferrari et al. 2014). Simulations of individual mosquito movement have been used to study biting rates between vectors and hosts (Cummins et al. 2012; Maneerat and Daudé 2016). These models were designed to study movement rates over limited spatial and temporal scales but do the nature of their design are unable to simulate movement at the population level (Cummins et al. 2012; Maneerat and Daudé 2016). Recently, a spatially explicit model has been applied to the spread of Ae. aegypti infected with Wolbachia under different levels of habitat quality and seasonal complexity (Hancock et al. 2018). Although the authors did not incorporate barriers to movement, the level of simulated population movement in mosquitoes ranged from $\sim 100$ to $400 \mathrm{~m}$ over 2 
years, reflecting the general low dispersal of $A e$. aegypti (Hancock et al. 2018). Simulating fine scale mosquito movement as part of a larger meta-population is relatively new and there are a number of knowledge gaps not addressed by previous studies. In particular, how barriers and landscape features such as the availability of larval habitat influence Ae. aegypti population connectivity and spread through urban landscapes.

Modelling approaches to addressing these issues are complex and intractable. Here we set out to use a spatially explicit network model, parameterized with movement and development data from the literature, to simulate the invasion and spread of Ae. aegypti among rainwater tanks in Brisbane. Our modelling approach focuses on the spread of a newly established mosquito population as it moves through the urban landscape, utilizing rainwater tanks as key larval habitat. In creating our network approach, we sought to understand what suburban characteristics influenced connectivity and thus population spread. Model scenarios were based on different rates of rainwater tank non-compliance (sealed/unsealed to the movement of mosquito adults) that are consistent with historical entomological surveys. Considering these landscape configurations, we sought to test the hypothesis that greater rates of population spread would occur in areas where higher levels of rainwater tank were non-compliant. We discuss methods for managing and impeding the risk of spread and establishment of Ae. aegypti through the management of rainwater tank non-compliance and provide insight into key biological parameters.

\section{Methods}

Entities, state variables and scales

The network model was written in Java and the Repast Simphony 2.4.0 toolkit for agent-based modelling (North et al. 2013). The model consists of rainwater tank agents within a spatially explicit urban landscape defined primarily by roads acting as barriers to movement. Only rainwater tanks agents that are noncompliant (unsealed) can become infected by the Ae. aegypti population. Local council datasets were used to identify rainwater tank locations (Fig. 1; Brisbane City Council 2012) and road locations from a state geospatial database (Supplemental F1; Queensland Government 2017). The model runs on a 2-week time step, within which mosquito populations and movement probabilities are calculated and populations reset equally across the landscape after every 6 months, to conservatively simulate the effect of the winter season and a retraction back into key larval habitat: rainwater tanks. The landscape is made up of Statistical Local Area (SLA) boundaries (referred to interchangeably as suburbs), the spatial measure used by the Australian government as political and statistical boundaries when the rainwater tank data was collated in 2011. For statistical analysis, each model replicate was run within selected SLAs of Brisbane, Australia, over five years, with perimeters of each SLA considered a discrete linear boundary (Fig. 1). It did not consider movement between SLAs such as random dispersal via human-mediated transport. A SLA would not be chosen for simulation if: the only tanks available were close to the spatial boundary, tanks within the suburb were separated by a large geographic distance, central tanks were isolated without chance of forming a link, or the total number of tanks in the suburb was less than 100. These included areas such as the central business district where results are uninformative. This resulted in the removal of 15 suburbs that were not suitable for modelling spread which tended to be inner-city areas where tanks were not present or larger peri-urban areas with residential developments on the periphery of the suburb boundary (Fig. 1).

Given Ae. aegypti tends to exhibit low dispersal, movement scenarios were selected to represent four levels of landscape connectivity, and from each SLA mosquito population spread was measured. High noncompliance rates (90\%, high connectivity) represent a scenario when regulations were first introduced into Brisbane 100 years ago (Elkington 1913); the half non-compliance scenario (50\%) represents the condition of rainwater tanks when the last large epidemic of dengue occurred in Brisbane during 1925/26 (Hamlyn-Harris 1927), a contemporary 'worst-case scenario' was selected at $30 \%$ non-compliance, and a low scenario (10\%, low connectivity) was chosen to represent the condition of tanks during the last known entomological surveys in Brisbane (Heersink et al. 2015). To ensure landscapes were generated stochastically, a compliance state (yes/no) was randomly assigned to each tank before running the model. The selection on the initial tank infested with mosquitoes 


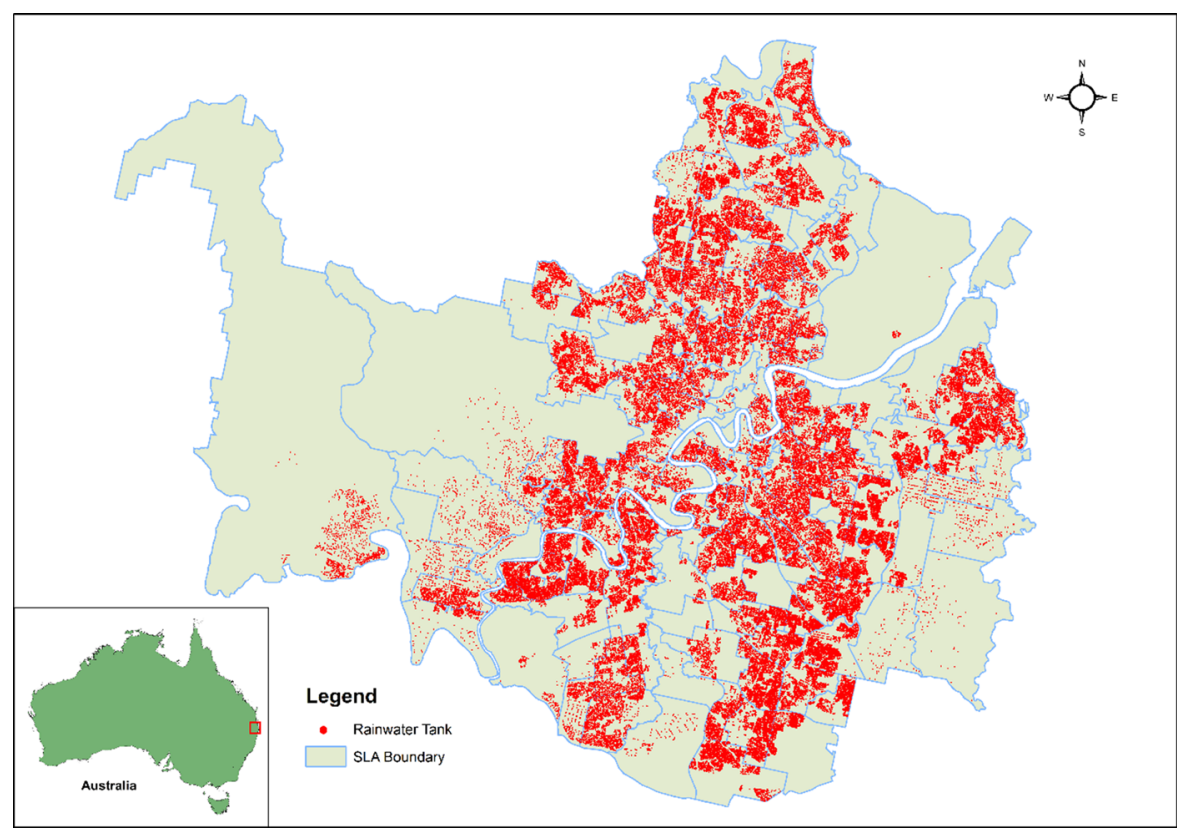

Fig. 1 The location of rainwater tanks (Brisbane City Council 2012) and Statistical Local Areas (SLA, or suburb) within Brisbane, Australia, used in modelling mosquito spread. Map

was chosen for each suburb; the first infected tank was defined as the closest tank to the SLA centroid. If this initial infestation was isolated $(>500 \mathrm{~m}$ from another point) or surrounded by main roads the next closest tank was selected until the restrictions no longer applied.

Network topology and movement

The processes occurring within the model are split into two parts; a 'population model' within each node (tank) and a 'movement behaviour' model representing links between nodes (Supplementary F2). The mosquito population in each node is a simple logistic growth population model (Kucharavy and De Guio 2015).

$\frac{d N}{d t}=r N\left(\frac{K-N}{K}\right)$

where $\mathrm{N}$ is the number of individuals in a population, $\mathrm{t}$ is time, $r$ is the maximum per capita growth rate and $\mathrm{K}$ is the carrying capacity (Table 1). The carrying capacity was set to less than the number of potential Ae. aegypti adults from the maximum larvae $(6,600$ larvae) observed in a rainwater tank by Tun-Lin et al. base layer sourced from Australian ABS digital boundary (Australian Bureau of Statistics 2016) and licensed under Creative Commons 2.5 (2016)

(1995). The rate of growth is based on a published Ae. aegypti population model where the simulation starts with ten egg laying females and reaches equilibrium at approximately 200 days (Dye 1984). An extinction threshold of one individual mosquito was incorporated into tanks to simulate population instability.

Movement within the network model takes the isotropic Gaussian dispersal kernel framework developed within Trewin et al. (2020) and we derive movement probabilities from the Ae. aegypti markrelease-recapture (MRR) dataset of Russell et al. (2005) from Cairns, Australia. From this dispersal kernel, the probability of mosquitoes moving from one rainwater tank to another was derived into a cumulative distribution function that considers all available rainwater tanks within the maximum distance, assuming unconstrained movement (see below, Maciel-deFreitas and Lourenço-de-Oliveira 2009). Within the current model, movement is based on diffusion through the landscape (Kareiva and Shigesada 1983; Zalucki et al. 2015). For an individual starting from the origin, the isotropic Gaussian probability density function (iGPDF) for the individual's position $(x, y)$ after time $t$ is implemented as: 
Table 1 Parameters and descriptions of Aedes aegypti and rainwater tank agents within the network simulation

\begin{tabular}{lll}
\hline Parameter & Values & Source \\
\hline $\begin{array}{l}\text { Movement parameters } \\
\text { Distance }\end{array}$ & iGPDF & $\begin{array}{c}\text { Developed from Russell et al. (2005) dataset and } \\
\text { extended from Trewin et al. (2020) } \\
\text { Russell et al. (2005) }\end{array}$ \\
$\begin{array}{l}\text { Average life expectancy }(\lambda) \\
\text { Time step }\end{array}$ & 7.1 days & \\
$\begin{array}{l}\text { Maximum distance of travel } \\
\text { Probability of crossing road }\left(p_{R}\right)\end{array}$ & $500 \mathrm{~m}$ & Maciel-de-Freitas and Lourenço-de-Oliveira (2009) \\
Probability of staying at a node $\left(p_{\text {stay }}\right)$ & 0.184 & Developed from Russell et al. (2005) \\
Agent parameters (rainwater tanks) & 0.086 & Developed from Russell et al. (2005) \\
Tank ID & $1-n$ tanks & \\
Infected tank & Infected or uninfected & \\
Compliance with regulations & Compliant or non-compliant & \\
$r$ (maximum per capita growth rate) & 0.4 & Dye (1984) \\
Carrying capacity $(K)$ & 350 & Dye (1984) \\
Tank spatial location & $x, y$ coordinate in space & Brisbane City Council (2012) \\
\hline
\end{tabular}

$f_{X, Y}(x, y \mid t)=\left(2 \pi \sigma^{2} t\right)^{-1 / 2} e^{\frac{-\left(x^{2}+y^{2}\right)}{\sigma^{2} t}}$

where $\sigma$ is the time varying standard deviation of the dispersal kernel (Trewin et al. 2020). This diffusion model is linked to the mark-recapture data by modelling individual counts of adult mosquitoes in traps, $C_{i}$, from a location $\left(x_{i}, y_{i}\right)$ over some interval $[a, b]$ as the outcome of a Poisson process with intensity function proportional to $\Lambda_{i}=p_{R}{ }^{r_{i}} \int_{a}^{b} f\left(x_{i}, y_{i} \mid t\right) d t$. Here, $p_{R}$ is the thinning that is applied to the Poisson process for each road that is present between the trap and the release location and $r_{i}$ is the minimum number of roads that must be crossed to reach the trap, so that

$C_{i} \sim \operatorname{Poisson}\left(k \Lambda_{i}\right)$

where $k$ is an empirical scaling parameter which relates to the relative trap counts to the integral of the Gaussian probability density function. The distance travelled within the iGPDF model is time dependent, thus the life expectancy will determine how far mosquitoes will move. This component of the model starts with estimating the average life expectancy for Ae. aegypti $(\lambda)$ from probability of daily survival (PDS) in MRR data from Cairns (Russell et al. 2005) using the methods of Niebylski and Craig Jr (1994) where $\lambda$ is $1 /-\log _{\mathrm{e}} \mathrm{PDS}$ (Table 1 ). The estimated average life expectancy (7.1 days) is within the range of those observed in other papers estimating $\mathrm{Ae}$. aegypti PDS (Maciel-de-Freitas et al. 2004; Muir and Kay 1998). As the distance travelled within the density function relies on how long an individual lives, female Ae. aegypti life spans were randomly sampled as $m=1,000,000$ life-times, $t_{1}, \ldots, t_{\mathrm{m}}$, using an exponential life-time PDF:

$f_{T}(t)=\frac{1}{\lambda} e^{-t / \lambda}$

These samples were used to randomly generate a set of movement coordinates $\left(x_{1}, y_{1}\right), \ldots,\left(x_{\mathrm{m}}, y_{\mathrm{m}}\right)$ using the probability density function in Eq. 2, conditional on each of the sampled life-times (i.e. we substitute each of the life-times into Eq. 2, for $t$ and then sample a location $(x, y)$ from the density). These coordinates were in turn used to generate the absolute travel distances for each sampled life-time, so that for the $i$ th coordinate, this was $d_{\mathrm{i}}=\left(x_{i}^{2}+y_{i}^{2}\right)^{1 / 2}$.

A probability mass function (PMF) was created by returning the absolute distance travelled at $5 \mathrm{~m}$ intervals for ease of reference within the network model (Supplementary F3). To estimate the probability of a link forming between two nodes in the network (facilitating two-way movement), an empirical cumulative distribution function (CDF; Supplementary F4) was integrated from the static PMF. As Ae. aegypti tend to avoid crossing roads, the Russell et al. (2005) dataset was used to estimate the probability of a mosquito crossing a road over a two-week period 
(Table 1). Roads were imported as shapefiles into the Repast context from Queensland state geospatial database of roads (Queensland Government 2017). For each link formed between nodes (tanks), the number of roads is counted along the link and the probability of mosquitoes moving across the road is modified by the number of roads they cross. Major roads are displayed within the Repast geography as two single parallel roads, thus halving the probability of mosquitoes crossing them.

Once links in the network had been generated, the proportion of the population leaving and staying at the current node was based on the equations:

$\pi\left(i, N_{j} \mid N\right)=\frac{\left(1-p_{\text {stay }}\right) p\left(i, N_{j}\right) \times p_{R}^{R\left(i, N_{j}\right)}}{\sum_{k=1}^{n} p\left(i, N_{k}\right) \times p_{R}^{R\left(i, N_{k}\right)}}$

And

$\pi(i, i)=p_{\text {stay }}$

where $i$ is a node that is a source of infestation, $N=$ $\left\{N_{1}, \ldots, N_{n}\right\}$ is a set of neighbouring nodes connected to $i$ that represent non-compliant tanks, of which $N_{j}$ is a member, $\pi\left(i, N_{j}\right)$ is the proportion of the population that move from node $i$ to node $N_{j}$ and $p_{\text {stay }}$ is the proportion of the population that stays at node $i$ (Table 1 , see description below). $p\left(i, N_{k}\right)$ is the unnormalised proportion moving the distance between node $i$ and $N_{k}$ in the absence of roads, $p_{R}$ is a parameter that represents the probability that a mosquito crosses a road, $R\left(i, N_{k}\right)$, the number of roads that must be crossed in order to move between node $i$ and node $N_{k}$. $p\left(i, N_{k}\right)$ was equal to the PMF obtained for the probability of travelling the distance between tank $i$ and neighbour $N_{k}$.

Once all potential links have formed to surrounding nodes, each mosquito at a node is randomly selected to stay at the node for this timestep (with probability $p_{\text {stay }}$ ) or to move to one of the neighbouring nodes (with probability $1-p_{\text {stay }}$ ). One the random number of moving mosquitoes has been established, fixed proportions of these individuals were then moved to neighbouring nodes, so that the proportion of moving mosquitoes that transitioned from node $i$ to neighbour $N_{j}$ was $\frac{\pi\left(i, N_{j} \mid N\right)}{1-p_{\text {stay }}}$. The parameter $p_{R}$ was calculated as $p_{R}=0.184$ (95\% Confidence Interval Upper 0.187, Lower 0.181). Using Eq. 5 for the observed data, the scaling parameter, $k$, the standard deviation, $\sigma$, and the road thinning parameter, $p_{R}$, can be estimated through maximum likelihood with optimization carried out via a Nelder-Mead simplex method via the optim() routine in R. Although $\sigma$ and $k$ are only applied when deriving the PMF, these were determined as 79.01 and 109.11 , respectively.

We defined $p_{\text {stay }}$ as the probability of a mosquito travelling at most half the length of a typical residential property in Cairns (approximately $20 \mathrm{~m}$ ). The proportion of the population staying within this distance is then defined by the CDF ( $p_{\text {stay }}$, Table 1; Supplemental F4). At the end of each time step in the model, each tank's mosquito population is updated to reflect the internal tank population increase, the mosquitoes that emigrated to a new tank, and those that immigrated to the current tank. Within the display network of the model, a link is formed between the two nodes where movement was successful. This process happens for each tank populated with mosquitoes at each time step.

\section{Outputs}

The three main outputs of the model at each time step were:

(1) whether a node (rainwater tank) has mosquitoes;

(2) the number of mosquitoes within each rainwater tank; and .

(3) the total number of links from a source rainwater tank to recipient tanks (out-links).

For each suburb and scenario of non-compliance the model was run 30 times. Mosquitoes infest noncompliant rainwater tanks (as nodes) as they move through the network. The complete network topology for each suburb is added to the Repast context for display at the end of each simulation step and all results were output to.csv files for analysis.

\section{Statistical analysis}

To mitigate the effect of the modifiable-areal-unit problem, results were reported at the largest areal unit available, in this case the suburb. The full list of covariates used in analysis were extracted using ArcGIS (ESRI 2017) at the suburb level and included total rainwater tanks, tanks per square kilometre, total area of suburb $\left(\mathrm{km}^{2}\right)$, total properties (lot and plan 
from cadastre), mean property size $\left(\mathrm{m}^{2}\right)$, properties per square kilometre, total cadastral plans, mean plan size $\left(\mathrm{m}^{2}\right)$, total roads, total road length $(\mathrm{km})$, roads per square kilometre, mean road length $\left(\mathrm{km}^{2}\right)$, total population, population density $\left(\mathrm{km}^{2}\right)$ and socio-economic decile (Supplemental T1). Correlation coefficients were calculated and predictors with values over 0.75 were removed to avoid collinearity (Supplemental F5). Datasets were provided by the (Brisbane City Council 2012), Australian Bureau of Statistics (Australian Bureau of Statistics 2011a) and the Queensland government (Queensland Government 2017).

The percentage, arithmetic mean and standard deviation of infested, non-compliant tanks, the mean number of non-compliant tanks and the maximum number of infested tanks were calculated for model runs over a 5-year period. To test which scenarios resulted in the largest number of infected tanks, oneway ANOVA was used to compare each of the four non-compliance scenarios across all suburbs. To satisfy the assumption of equal variance, the numbers of infected tanks were square root transformed, replications were reduced to 10 to reduce the degrees of freedom in the analysis, and Tukey's honestly significant difference test was used for multiple comparisons between non-compliance scenarios.

To determine which suburbs represented the highest risk of mosquito spread through non-compliant rainwater tanks, analyses were performed on model outputs. To determine which suburbs facilitated mosquito spread, model outputs were chosen to represent the $90 \%$ rainwater tank non-compliance scenario. This scenario represented the highest potential for mosquito spread between nodes. As the size of a mosquito population within each suburb was highly correlated with the number of rainwater tanks containing mosquitoes, the number of infested tanks was chosen for analysis. The total and mean number of infested rainwater tanks per suburb (over 30 replicated five-year simulations, rounded to the nearest integer) were calculated. Due to over dispersion in infested rainwater tank counts across different suburbs, where the variance was considerably larger than the mean, a generalized linear model with negative binomial errors for prediction was applied. The glm.nb() with a log link function 'MASS' library for R was applied during analysis. Residuals were normally distributed and a forward selection methodology was applied to choose optimal predictors, improve model fit and minimize AIC. Model predictions were used to identify which suburbs contained the greatest mean number of infested rainwater tanks.

To estimate the rate of mosquito spread across different compliance scenarios we selected (in this case total infested tanks over 5 years), ten high ( $>90$ th percentile), medium (50th percentile) and low risk ( $<$ 10th percentile) suburbs as predicted by the negative binomial model. Replicates were extracted from each suburb for the $10 \%, 30 \%$ and $90 \%$ noncompliance scenarios. The spread profiles of each suburb as predicted were ranked into quartiles, displayed using ArcGIS and ten suburbs with the highest and lowest spread were highlighted (see results Fig. 3).

To predict which suburbs consistently had the highest connectivity or "hubs" within the tank network, we derived the maximum number of outlinks for each suburb, per compliance scenario, and model replicate. Multiple regression was then applied to maximum out-links for the $90 \%$ rainwater tank noncompliance scenario, as this provided the greatest potential for nodes with high connectivity. No transformation of the dependant variable was necessary as residuals were normally distributed and the $\operatorname{lm}()$ function was applied from the base $\mathrm{R}$ package for analysis.

A forward selection methodology was applied to choose predictors and improve model fit using the multiple $\mathrm{R}^{2}$ value. We considered independent variables to be significant at the $P=0.05$ level for all models. To visualize connectivity within selected areas from high, medium and low risk suburbs (as predicted by the infested tanks risk statistical model) heat maps were created in ArcGIS using the kernel density tool within the Spatial Analyst toolset. This tool was applied to visualize groups of nodes within the landscape with high mean out-links after five years. Output cell size was set to one metre, search radius $75 \mathrm{~m}$, with output values as densities and the planar method was applied.

To understand the area that a mosquito invasion may spread through the urban landscape, suburbs from high moderate and low risk categories were selected. Fifteen additional replicate invasions proceeded for five years under each scenario of non-compliance within the model. The area of spread across the landscape, measured as an ellipse, was used to give an estimated area of spread for each compliance scenario. 
As widths and heights of invasions were never constant, the mean ellipse area was calculated for fifteen iterations of each model run in $\mathrm{km}^{2}$. As the assumptions of ANOVA were not met, a KruskalWallis test and pairwise Wilcox test with Bonferroni correction were used for group and pairwise comparisons, respectively. All analyses were performed in R v3.5.1 (R Core Team 2018).

\section{Results}

Infested rainwater tanks

There were significantly higher infestations in the $90 \%$ non-compliance scenario when compared with all other scenarios $(\mathrm{F}(3,36)=17,495, p<0.001)$. A post hoc Tukey test revealed significant differences in mean infestation level between all paired scenarios. Interestingly, the 50\% non-compliance scenario represented the highest percentage of infested tanks when compared with other scenarios (Table 2). The $10 \%$ non-compliance scenario represented a lower percentage and mean number of infested tanks than all other scenarios (Table 2). Results of the negative binomial model predicting total infested rainwater tanks showed a three-way interaction between the covariates; tanks per $\mathrm{km}^{2}$, the mean size of a property and the mean road length of suburbs (Table 3).

\section{Maximum out-links and connectivity}

To understand which landscapes promoted the highest mosquito population connectivity, nodes that were most frequently represented in the top $1 \%$ of nodes with high out-links (within the $90 \%$ non-compliance scenario) were examined. Of these "hubs" with total out-links of between 9 and 18,15\% were primarily identified as nodes where the original invasion began. After thirty replications these nodes represented $48 \%$ of all nodes with high out-degrees in the top $1 \%$ of hubs. Heat maps revealed groups of nodes within the landscape with high connectivity (Fig. 2). Low risk suburbs (Fig. 2 A/B) had considerably lower connectivity and area of spread than medium (Fig. 2 C/D) and high-risk suburbs (Fig. 2 E/F). Mosquito population connectivity was best explained in suburbs by the interaction of high tank density, small yard size and high human population density (Table 4).
The total number of infested tanks were used to estimate and display which suburbs within Brisbane represent the highest risk of spread. In the $90 \%$ noncompliance scenario, the high-spread suburbs tended to be predicted by a large densities of rainwater tanks, followed by the mean area of residential properties, and these suburbs tended to be aggregated spatially within Brisbane (Fig. 3).

Population spread

Once suburbs representing the highest areas of population spread were identified through replication, the model was additionally run to simulate five years and the area of invasion under each non-compliance scenario measured. The greatest area of spread was observed in the $50 \%$ non-compliance scenario, with a mean area of $0.842 \mathrm{~km}^{2}(\mathrm{SD} \pm 0.096)$, followed by the $30 \% \quad\left(\right.$ mean $\left.=0.722 \mathrm{~km}^{2}, \quad \mathrm{SD} \pm 0.152\right), \quad 90 \%$ $\left(0.703 \mathrm{~km}^{2}, \mathrm{SD} \pm 0.088\right)$ and $10 \%\left(0.216 \mathrm{~km}^{2}, \mathrm{SD} \pm\right.$ 0.031) scenarios, respectively (Fig. 4). There were significant differences between non-compliance scenarios within suburbs of high $(\mathrm{H}(3)=42.2$, $P=<0.001), \quad$ moderate $\quad(\mathrm{H}(3)=36.5$, $P=<0.001)$ and low $(\mathrm{H}(3)=44.9, P=<0.001)$ spread. However, pairwise comparisons revealed no significant differences between the $30 \%$ and $90 \%$ noncompliance scenarios in the high and moderate spread suburbs and between the $30 \%$ and $50 \%$ scenarios in the moderate spread suburb (Fig. 4). There were significant differences between suburbs when comparing the effect of non-compliance on population spread in the $90 \%(\mathrm{H}(2)=16.4, P=<0.001), 50 \%$ $(\mathrm{H}(2)=26.0, \quad P=<0.001), \quad 30 \% \quad(\mathrm{H}(2)=30.4$, $P=<0.001)$ and $10 \%(\mathrm{H}(2)=25.9, P=<0.001)$ scenarios, respectively. Pairwise comparisons revealed no significant difference between high and medium risk suburbs in the $90 \%, 30 \%$ and $10 \%$ noncompliance scenarios.

\section{Discussion}

Rainwater tanks represent ideal mosquito larval habitat and non-compliant tanks can be a key driver of mosquito establishment, spread, and subsequent disease transmission (Trewin et al. 2017). Our findings suggest that although mosquito population spread within the landscape is enhanced when tank density 
Table 2 Descriptive statistics of model outputs including the mean number of infested tanks, mean number of non-compliant tanks and the maximum number of infested tanks over 5 years under different rainwater tank non-compliance scenarios

\begin{tabular}{lllll}
\hline $\begin{array}{l}\text { Non-compliant rainwater tank } \\
\text { scenarios }(\%)\end{array}$ & $\begin{array}{l}\text { Mean infested non-compliant } \\
\text { tanks }(\%)\end{array}$ & $\begin{array}{l}\text { Standard } \\
\text { deviation }\end{array}$ & $\begin{array}{l}\text { Mean non-compliant } \\
\text { tanks }\end{array}$ & $\begin{array}{l}\text { Max \# infested } \\
\text { tanks }\end{array}$ \\
\hline 10 & $5.8(12.2)$ & 0.4 & 63.6 & 22 \\
30 & $29.4(18.9)$ & 0.6 & 190.6 & 93 \\
50 & $52.5(21.0)$ & 1.3 & 317.9 & 160 \\
90 & $82.8(19.1)$ & 0.7 & 571.7 & 210 \\
\hline
\end{tabular}

Table 3 Results of negative binomial analysis with coefficients predicting the simulated number of infested tanks (90\% noncompliance scenario) over 5 years from 140 suburbs within Brisbane.

\begin{tabular}{|c|c|c|c|c|}
\hline Coefficients & Estimate & Std Error & $\mathrm{z}$ value & $P$ value \\
\hline Intercept & 4.191 & 0.344 & 12.179 & $<0.001$ \\
\hline Tanks $/ \mathrm{km}^{2}$ & -0.002 & 0.003 & -0.863 & 0.388 \\
\hline Mean $(\bar{x})$ property size $\left(\mathrm{km}^{2}\right)$ & -78.198 & 2.830 & -0.713 & 0.476 \\
\hline Mean road length $(\mathrm{km})$ & -2.019 & 26.584 & -2.942 & $0.003 *$ \\
\hline Tanks $/ \mathrm{km}^{2}$ : Mean road length & 0.029 & 0.024 & 1.226 & 0.220 \\
\hline Mean road length: Mean property size & 167.331 & 67.766 & 3.744 & $<0.001 *$ \\
\hline Tanks $/ \mathrm{km}^{2}$ : Mean property size & 4.637 & 1.239 & 2.469 & $0.014^{*}$ \\
\hline Tanks/km ${ }^{2}: \bar{x}$ Road length: $\bar{x}$ Property size & -21.841 & 9.664 & -2.26 & $0.024 *$ \\
\hline
\end{tabular}

Null deviance: 274.99 on 139 degrees of freedom

Residual deviance: 132.50 on 132 degrees of freedom

AIC: 681.65

Number of Fisher Scoring iterations: 1

Theta: 12.99

Std. Err. 4.85

$2 \times$ log-likelihood: -663.65

*indicates significance at $P<0.05$

and non-compliance rates increase, this relationship is not linear and only really evident when compliance is lower than $30 \%$. We observed a rapid increase in the area of populations between the 10 and $30 \%$ scenarios before spread plateaued at higher levels of noncompliance. This suggests there may be a threshold of non-compliance that dramatically affects the spread of a population within urban environments.

To lower the risk of establishment and spread of invasive mosquitoes like Ae. aegypti, it will be important to maintain a high level of rainwater tank regulatory compliance within major Australian cities. Within the $10 \%$ non-compliance scenario, larval habitat was highly fragmented and due to the low dispersal of female Ae. aegypti, population spread was limited. It is interesting to note that non-compliance levels of $\sim 10 \%$ were recorded in Brisbane around the time Ae. aegypti was driven to extinction in the mid-1900s (Trewin et al. 2017) and rainwater tank non-compliance levels of $<10 \%$ do not correlate with the native container inhabiting mosquito, Ae. notoscriptus, distribution or larval abundance (Heersink et al. 2015). Furthermore, it is possible that noncompliance rates under a $10 \%$ threshold could lower the risk of establishment and spread of a newly invading population, particularly if sealed tanks are acting as population sinks (Trewin 2018). Ensuring tanks remain sealed could act much like the 

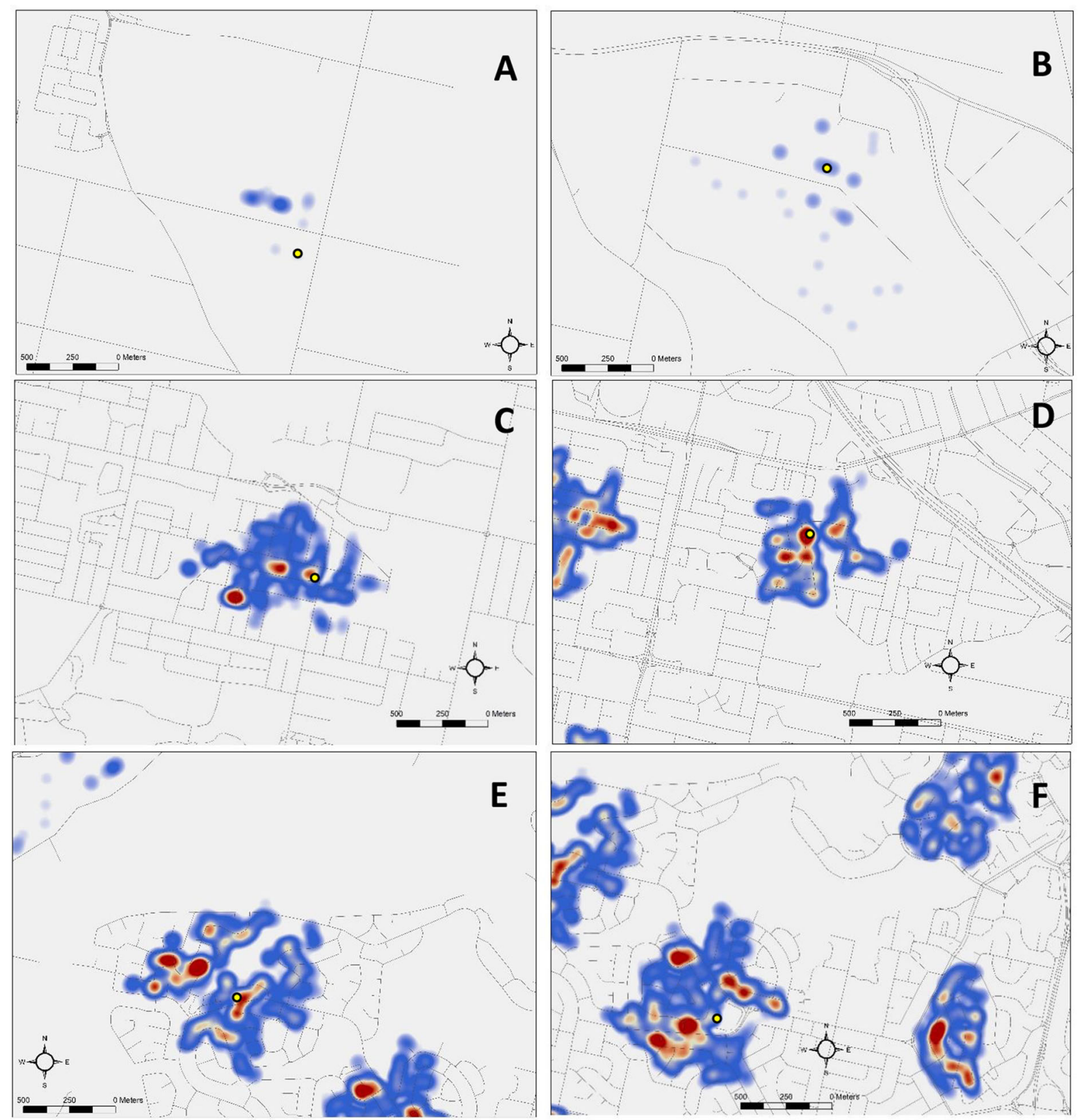

Fig. 2 Heat maps representing connectivity (mean out-links) of nodes within low (a, b), medium (c, d) and high (e, f) risk SLAs under a $90 \%$ non-compliance scenario. Yellow circle represents node of initial infestation

epidemiological theory called the "mass-action principle" and is the theory underlying herd immunity (Fine 1993). This principle would suggest that infestation frequency is related to the product of proportion infested multiplied by the number of non-compliant tanks (Paul 1979). It would be prudent if authorities could limit non-compliance levels to less than $10 \%$, particularly in suburbs which are at high risk for establishment and spread.

It was expected that the area of mosquito population spread would be highest in suburbs with a $90 \%$ rainwater tank non-compliance level. However, we observed the greatest level of spread within $30 \%$ and $50 \%$ non-compliance scenarios. There are likely two 
Table 4 Results of multiple regression analysis comparing the maximum number of simulated out-links in each SLA (or suburb; $90 \%$ non-compliance scenario) over 5 years within Brisbane

\begin{tabular}{lllrr}
\hline Coefficients & Estimate & Std. error & t value & $P$ value \\
\hline Intercept & 5.64 & $1.60 \mathrm{E}-01$ & 35.36 & $<0.001$ \\
Tanks $/ \mathrm{km}^{2}$ & $1.27 \mathrm{E}-02$ & $9.66 \mathrm{E}-04$ & 13.09 & $<0.001$ \\
Mean $(\bar{x})$ property size $/ \mathrm{km}^{2}$ & $-6.11 \mathrm{E}+01$ & 4.56 & -13.40 & $<0.001$ \\
Human population $/ \mathrm{km}^{2}$ & $2.64 \mathrm{E}-05$ & $5.85 \mathrm{E}-05$ & 0.45 & 0.65 \\
$\bar{x}$ Propertysize:tanks $/ \mathrm{km}^{2}$ & 3.570 & $5.86 \mathrm{E}-01$ & 6.10 & $<0.001$ \\
$\bar{x}$ Propertysize:population $/ \mathrm{km}^{2}$ & $-1.79 \mathrm{E}-01$ & $3.79 \mathrm{E}-07$ & -3.64 & $<0.001$ \\
Population $/ \mathrm{km}^{2}:$ tanks $/ \mathrm{km}^{2}$ & $-1.38 \mathrm{E}-06$ & $4.40 \mathrm{E}-02$ & -4.06 & $<0.001$ \\
Tanks $/ \mathrm{km}^{2}: \bar{x}$ propertysize: population $/ \mathrm{km}^{2}$ & $1.34 \mathrm{E}-03$ & $3.92 \mathrm{E}-04$ & 3.41 & $<0.001$ \\
\hline
\end{tabular}

Residual standard error: 2.11 on 4166 degrees of freedom

Multiple R-squared: 0.37, Adjusted R-squared: 0.37

F-statistic: 354.8 on 7 and $4166 \mathrm{DF}, P$ value: $<0.001$

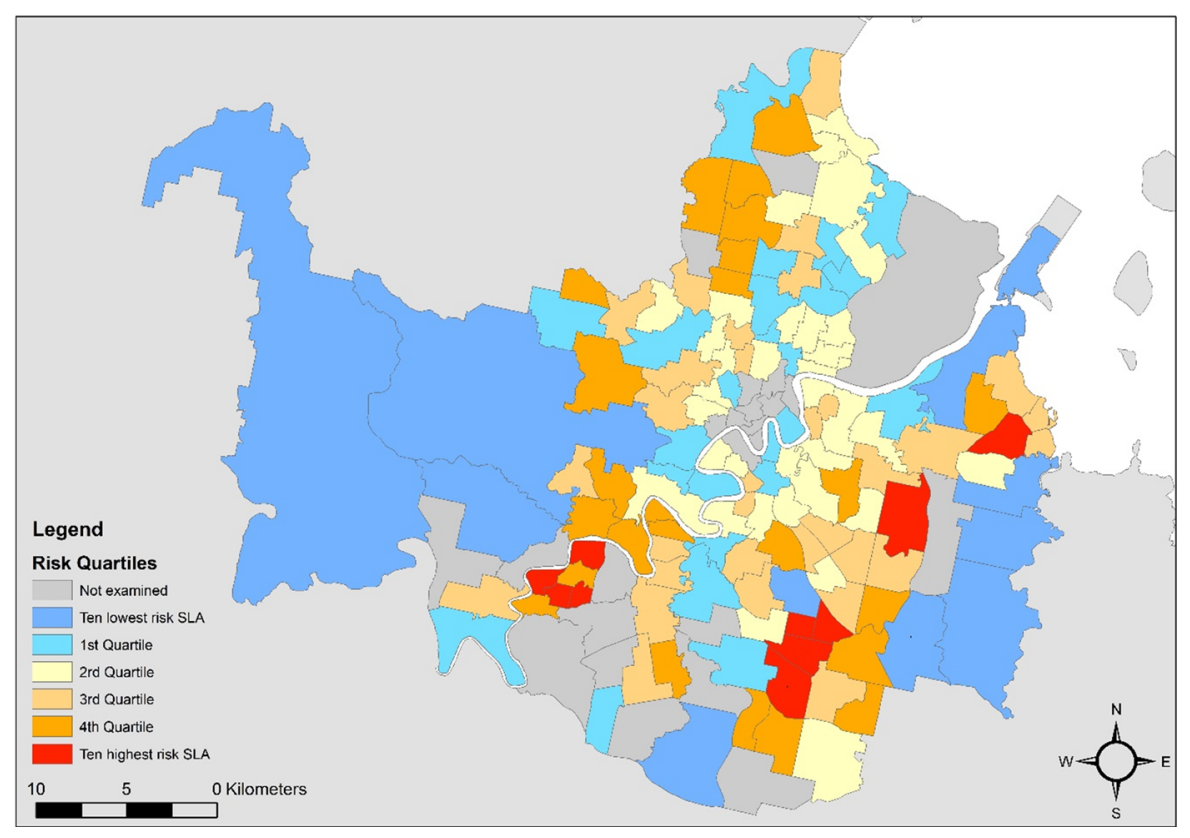

Fig. 3 Map indicating which Statistical Local Areas represent where high (red), moderate (yellow) and low (dark green) spread of mosquitoes would occur under a $90 \%$ rainwater tank non-

reasons for why this may have occurred. First, distances between non-compliant tanks would be greater in the $30 \%$ and the $50 \%$ non-compliance scenarios relative to the $90 \%$ scenario, but not so far that mosquitoes consistently die when attempting movement (such as would be the case in the $10 \%$ noncompliance scenario). One mechanism to explain this could be related to the probability of movement within compliance scenario. For increased visualization of low/high spread, we have included the ten highest and lowest risk SLAs as separate colours

the PMF, where the probability of movement does not vary greatly between 20 and $90 \mathrm{~m}$, allowing for large jumps where possible. A second mechanism may relate to the $30 \%$ and $50 \%$ non-compliance scenarios observing a greater number of mosquitoes moving to a smaller number of potential nodes. With $20 \%$ of mosquitoes attempting to disperse staying at the original infected tank (under $20 \mathrm{~m}$ ) and the remaining 


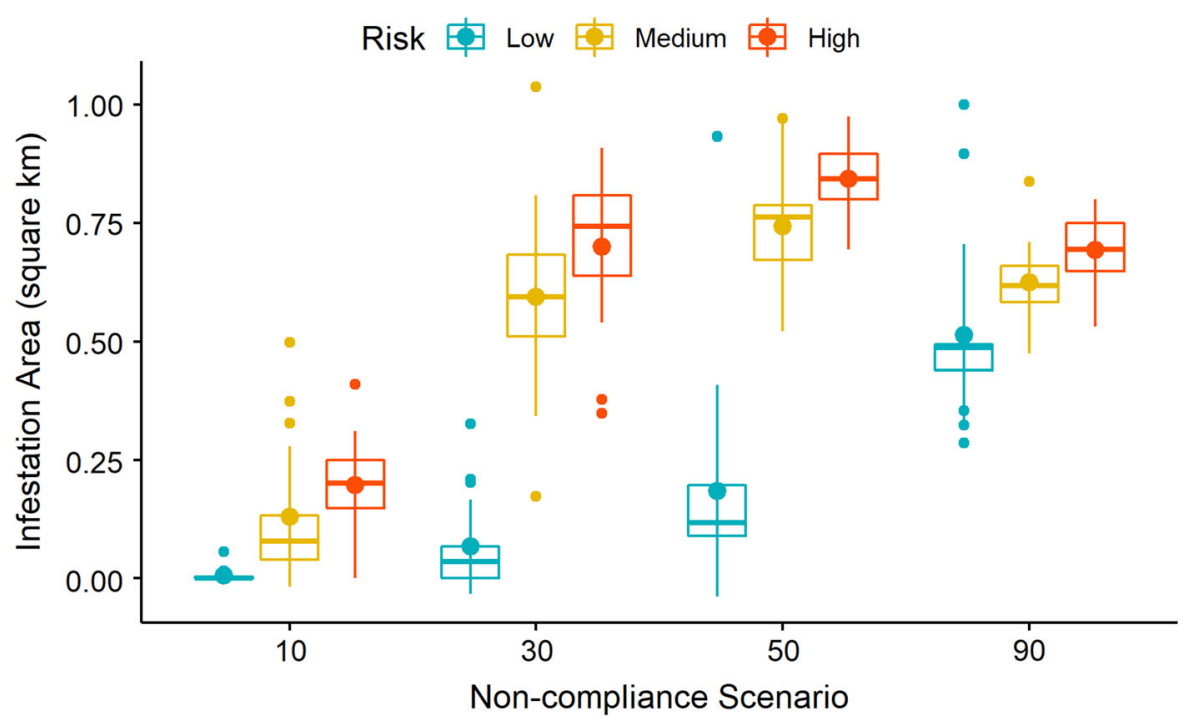

Fig. 4 Plot of invasion area $\left(\mathrm{km}^{2}\right)$ for each scenario of noncompliance $(10 \%, 30 \%, 50 \%$ and $90 \%)$ in suburbs identified to contain a high (red), medium (orange) and low (blue) risk of

$80 \%$ attempting to disperse over distances greater this this, the number of mosquitoes distributed into nearby available non-compliant tanks depends on the number of links formed. A large number of links forming to infected tanks close to the tank of origin would result in small numbers of individuals moving between tanks-a function of the dispersing population, the number of links formed, and how the $80 \%$ attempting to disperse are split between dispersing links. However, a low number of connected nodes may lead to larger number of mosquitoes successfully 'jumping' between nodes, initiating destination populations with a greater number of individuals. In this case, populations in newly infested nodes would increase more rapidly when colonized by a larger number of mosquitoes, thus allowing for larger dispersal events through the landscape. A combination of these mechanisms may have enhanced the spread of populations within the model landscape at lower rainwater tank non-compliance levels.

When landscape conditions are sub-optimal, mosquitoes may need to move further to seek out favourable habitat. This has been supported by modelling by Otero et al. (2008) and observed in field experiments (Bugher and Taylor 1949; Honório et al. 2009; Wolfinsohn and Galun 1953). Otero et al. (2008) suggest that in areas of sub-optimal habitat (such as temperate areas), populations of Ae. aegypti may spread over a period of 5 years. Large points represent mean and error bars one standard deviation

prevent local extinction by dispersing over larger distances to new habitat. Thus, when population sources are sparsely distributed through a landscape, it may act to increase the distance of mosquito dispersal and movement. We have not included any lingering effects of sub-optimal periods such as overwintering or when a tank infection goes extinct. This is primarily due to the highly variable rate at which populations re-emerge due to climatic factors or mosquito control processes which are not considered in the current model. Our results support the assertion that dispersal is driven by the availability (or density) of oviposition sites (Reiter et al. 1995; Wolfinsohn and Galun 1953). Conversely, when conditions are favourable within the landscape, such as in the $90 \%$ non-compliance scenario, Ae. aegypti is unlikely to move large distances from where adults emerge (Edman et al. 1998; Harrington et al. 2005). Although spread was still relatively high within this scenario, it did not result in the largest area of invasion. It seems that mosquitoes in the $90 \%$ non-compliance scenario were more likely to move within a group of tanks than make large jumps, thus lowering the speed of spread across the landscape. These results may be a limitation to our model in its current form, as it is possible that human mediated transport (which was not tested within the model) at high mosquito densities could 
increase the rate of population spread across barriers and unfavourable habitat (Benedict et al. 2007).

In Brisbane, the prevalence of rainwater tanks in households is currently greater than $40 \%$ in many suburbs, which is our primary predictor of increased spread. Simulated infested tanks were used to understand how spatial covariates associated with each suburb can predict the models estimate of mosquito spread between tanks and allow for a comparison of suburbs with different risk profiles. As one would expect, our results suggest that higher rates of population spread are driven primarily by the density of non-compliant rainwater tanks in the landscape. Ideally, surveillance of rainwater tanks and mosquito vectors should target these high-risk suburbs. While some of the predictors used in statistical analysis were not directly included in the network model, the spatially explicit network topology we derived from real-world data can be considered a function of a larger number of empirical covariates. These covariates (eg. property size and population density) would therefore affect link formation indirectly and were used in analyses. Furthermore, road width is likely to vary both within and between landscapes and as such, recommendations would benefit from sensitivity testing of parameters, in particular the influence of roads on population spread over time.

Simulations show that high tank density, moderateto-large property size and small road lengths resulted in the largest number of infested tanks. Suburbs with a high tank density are likely to represent areas of residential housing further than $7 \mathrm{~km}$ from the centre of the city, have the space to contain a rainwater tank and require greater volumes of water for garden use. Within Brisbane, these areas represent residential neighbourhoods with a mean property size of $\sim 800-1,000 \mathrm{~m}^{2}$, which were settled primarily during the 1970s and $80 \mathrm{~s}$, and are dominated by culde-sac designs. In these suburbs, blocks tend to be shaped with the landscape, thus increasing the number of houses that can fit into oddly shaped tracts (eg. to the edge of rivers) such as the suburb of Westlake in western Brisbane. A non-uniform block shape enabled higher connectivity within the landscape and increased spread without forcing populations to cross roads to invade new habitat. The design of these urban environments provides a continuous space for population spread before individuals were required to cross a road and establish another infestation (Fig. 2).
Although these landscapes represent the highest rates of spread, they may not increase risk of establishment as modern house designs in these suburbs incorporate window screening and ducted air-conditioning which have been shown to influence disease transmission and vector abundance (Ramos et al. 2008; Reiter et al. 2003).

Our modelling suggests that all scenarios with a non-compliance rate over $30 \%$ had a similar influence on the rate of mosquito spread in suburbs. While our results focus on the random variation of non-compliant rainwater tanks between suburbs, it is likely that distributions of key larval habitats within suburbs (such as lower socio-economic areas) will vary across large cities. Future applications of this modelling framework should consider this intra-suburb variation on the rate of invasion spread at finer spatial scales. Our findings are conservative, however, as it was assumed the species could not persist long-term in Brisbane until recently (Trewin et al. 2019). Over a 5-year period, invasions extended to a radius of approximately $500 \mathrm{~m}$ and area of $0.7-0.9 \mathrm{~km}^{2}$, a conclusion which concurs with results from other modelling approaches with seasonal variation (Hancock et al. 2018). Aedes aegypti has not been observed in the city for over 60 years, despite ongoing city-wide surveillance and small populations found just $150 \mathrm{~km}$ to the north. Our findings may have implications for the ability to detect an incursion of Ae. aegypti within Brisbane and may require surveillance with a fine resolution if authorities are to detect an invasion early. If Brisbane were divided into square kilometre units (roughly the area estimated by the model that an $A e$. aegypti population would spread after 5 years) then one would need to monitor 16,000 ovitraps a year (1 trap per $\mathrm{km}^{2}$ ) to be certain of no establishment across the city. Unfortunately, surveillance of this magnitude is not always an efficient use of resources, both in terms of the time taken to deploy traps and manually identify specimens. However, with a combination of citizen science and the number of highly efficient, sensitive and cheaper genetic testing, detecting incursions of Ae. aegypti at the scale of a city may soon be possible (Montgomery et al. 2017). 


\section{Conclusion}

High rainwater tank non-compliance influences the rate at which Ae. aegypti populations spread through an urban landscape. Aedes aegypti is one of the most invasive species of disease vectors, as it is highly adapted for urban environments. Even low levels of non-compliance may rapidly increase the risk of establishment and spread of the species in suburbs containing high densities of rainwater tanks. This can be exacerbated when rainwater tanks are more highly connected, such as suburbs where the landscape is dominated by a cul-de-sac design. In the absence of any appropriately scaled surveillance program in Brisbane, and given the dispersal parameters of the vector, incursions are likely to go undetected. Governments and communities want to avoid the establishment of invasive vectors such as Ae. aegypti and Ae. albopictus (Skuse) and subsequent spread of dengue, chikungunya and Zika viruses. With the installation of over 300,000 rainwater tanks over the past 16 years and current non-compliance levels in Brisbane likely to be between 10 and 30\% (Brian Montgomery, Queensland Health, pers. comm.), rainwater tanks may be approaching ideal conditions for re-establishment and rapid spread of invasive vectors. Our results inform areas to target for future management actions in south-east Queensland and provide a pre-emptive approach that supports planning by health authorities for targeted surveillance for invasive mosquito vectors.

Funding This study was funded by an Integrated National Resources and Management grant from the University of Queensland and the Commonwealth Scientific and Industrial Research Organisation as part of a Doctorate Degree.

Availability of data and material(data transparency) The datasets generated during and analysed during the current study are available from the corresponding author on reasonable request.

Code availability(software application or custom code) The code created during the current study are available from the corresponding author on reasonable request.

\section{Declarations}

Conflict of interest The authors have no relevant financial or non-financial interest to disclose.

\section{References}

Australian Bureau of Statistics (2011a) 2011 Australian census data. http://www.abs.gov.au/websitedbs/censushome.nsf/ home/historicaldata2011?opendocument\&navpos= 280\&navpos $=75 \&$ navpos $=79$. Accessed 2 January 2017.

Australian Bureau of Statistics (2011b) Socio-Economic indexes for areas. Canberra.

Australian Bureau Of Statistics (2016) Australian statistical geography standard (ASGS): Volume 1-main structure and greater capital city statistical areas.

Benedict MQ, Levine RS, Hawley WA, Lounibos LP (2007) Spread of the tiger: global risk of invasion by the mosquito Aedes albopictus. Vector-Borne Zoonotic Dis 7:76-85

Brisbane City Council (2012) Rainwater tank database <electronic database $>$, Brisbance City Council Records, Brisbane. Accessed 20 October 2016 .

Bugher JC, Taylor M (1949) Radiophosphorus and radlostrontium in mosquitoes. Preliminary Report. American Association for the Advancement of Science, pp 146-7

Creative Commons 2.5 (2016) Creative commons license 2.5. https://creativecommons.org/licenses/by/2.5/. Accessed 20 October 2016.

Cummins B, Cortez R, Foppa IM, Walbeck J, Hyman JM (2012) A spatial model of mosquito host-seeking behavior. PLoS Comput Biol 8(5):e1002500. https://doi.org/10.1371/ journal.pcbi. 1002500

Dyck VA, Hendrichs J, Robinson AS (2006) Sterile insect technique: principles and practice in area-wide integrated pest management. Springer, Taylor \& Francis, Boca Raton.

Dye C (1984) Models for the population dynamics of the yellow fever mosquito, Aedes aegypti. J Anim Ecol 53:247-268

Edman JD, Scott TW, Costero A, Morrison AC, Harrington LC, Clark GG (1998) Aedes aegypti (Diptera: Culicidae) movement influenced by availability of oviposition sites. J Med Entomol 35:578-583

Elkington JSC (1913) Annual report of the commissioner of public health to 30th June. Office of the Commissioner of Public Health, Brisbane

ESRI (2017) ArcGIS Desktop. Release 10.4 ed. Redlands. CA: Environmental Systems Research Institute

Fahrig L, Merriam G (1985) Habitat patch connectivity and population survival. Ecology 66:1762-1768. https://doi. org/10.2307/2937372

Ferrari JR, Lookingbill TR, Neel MC (2007) Two measures of landscape-graph connectivity: assessment across gradients in area and configuration. Landscape Ecol 22:1315-1323

Ferrari JR, Preisser EL, Fitzpatrick MC (2014) Modeling the spread of invasive species using dynamic network models. Biol Invasions 16:949-960. https://doi.org/10.1007/ s10530-013-0552-6

Fine PEM (1993) Herd immunity: history, theory, practice. Epidemiol Rev 15:265-302

GorgasGorgas WC (1915) Sanitation in Panama. Appleton and company, New York and Londo. Vol 4, p. 1., 297, [1] p. D

Gubler DJ, Clark GG (1994) Community-based integrated control of Aedes aegypti: a brief overview of current programs. Am J Trop Med Hyg 50(6):50-60. https://doi.org/ 10.4269/ajtmh.1994.50.50 
Hamlyn-Harris R (1927) Mosquito Campaign 1927. Brisbane City Council, Brisbane.

Hamlyn-Harris R (1931) The elimination of Aedes argenteus Poiret as a factor in dengue control in Queensland. Liverpool Sch Trop Med 25(1):21-29

Hancock PA, Ritchie SA, Koenraadt CJ, Scott TW, Hoffmann AA, Godfray HCJ (2019) Predicting the spatial dynamics of Wolbachia infections in Aedes aegypti arbovirus vector populations in heterogeneous landscapes. J Appl Ecol 56(7):1674-1686

Harrington LC et al (2005) Dispersal of the dengue vector Aedes aegypti within and between rural communities. Am J Trop Med Hyg 72:209-220

Heersink DK, Meyers J, Caley P, Barnett G, Trewin B, Hurst T, Jansen C (2015) Statistical modeling of a larval mosquito population distribution and abundance in residential Brisbane. J Pest Sci 89:1:267-279. https://doi.org/10.1007/ s10340-015-0680-0

Hoffmann AA, Montgomery BL, Popovici J, Iturbe-Ormaetxe I, Johnson PH, Muzzi F, Greenfield M, Durkan M, Leong YS, Dong Y, Cook H, Axford J, Callahan AG, Kenny N, Omodei C, McGraw EA, Ryan PA, Ritchie SA, Turelli M, O'Neill SL (2011) Successful establishment of Wolbachia in Aedes populations to suppress dengue transmission. Nature 476(7361):454-457. https://doi.org/10.1038/ nature 10356

Honório NA, Codeço CT, Alves F, Magalhães MdAFM, Lourenço-de-Oliveira R (2009) Temporal distribution of Aedes aegypti in different districts of Rio de Janeiro, Brazil, measured by two types of traps. J Med Entomol 46:1001-1014

Kareiva PM, Shigesada N (1983) Analyzing insect movement as a correlated random walk. Oecologia 56:234-238. https:// doi.org/10.1007/bf00379695

Kucharavy D, De Guio R (2015) Application of logistic growth curve. Procedia Eng 131:280-290. https://doi.org/10.1016/ j.proeng.2015.12.390

Lumley GF, Taylor FH (1943) Part 1. Dengue. Part 2. Entomological. Sch publ Hlth Trop Med. Dep Hlth Aust 3:171

Maciel-de-Freitas R, Gonçalves JM, Lourenço-de-Oliveira R (2004) Efficiency of rubidium marking in Aedes albopictus (Diptera: Culicidae): preliminary evaluation on persistence of egg labeling, survival, and fecundity of marked female. Mem Inst Oswaldo Cruz 99:823-827

Maciel-de-Freitas R, Lourenço-de-Oliveira R (2009) Presumed unconstrained dispersal of Aedes aegypti in the city of Rio de Janeiro. Brazil Revista De Saúde Pública 43:8-12

Maneerat S, Daudé E (2016) A spatial agent-based simulation model of the dengue vector Aedes aegypti to explore its population dynamics in urban areas. Ecol Model 333:66-78. https://doi.org/10.1016/j.ecolmodel.2016.04. 012

Moglia M, Walton A, Sharma AK, Tjandraatmadja G, Gardner J, Begbie D (2013) Management of urban rainwater tank lessons and findings from south-east Queensland. Water 40:1-5

Montgomery BL, Shivas MA, Hall-Mendelin S, Edwards J, Hamilton NA, Jansen CC, McMahon Jamie L, Warrilow David, van den Hurk AF (2017) Rapid Surveillance for vector presence (RSVP): development of a novel system for detecting Aedes aegypti and Aedes albopictus. PLoS
Negl Trop Dis 11:e005505. https://doi.org/10.1371/ journal.pntd.0005505

Muir LE, Kay BH (1998) Aedes aegypti survival and dispersal estimated by mark-release-recapture in northern Australia. Am J Trop Med Hyg 58:277-282

Niebylski M, Craig G Jr (1994) Dispersal and survival of Aedes albopictus at a scrap tire yard in Missouri. J Am Mosq Control Assoc 10:339-343

North MJ, Collier NT, Ozik J, Tatara ER, Macal CM, Bragen M, Sydelko P (2013) Complex adaptive systems modeling with repast simphony. Complex Adapt Sys Model 1:3. https://doi.org/10.1186/2194-3206-1-3

Otero M, Schweigmann N, Solari HG (2008) A stochastic spatial dynamical model for Aedes Aegypti. Bull Math Biol 70:1297. https://doi.org/10.1007/s11538-008-9300-y

Paul EMF (1979) John Brownlee and the measurement of infectiousness: an historical study in epidemic theory. J Royal Stat Soc Series A (general) 142:347-362. https:// doi.org/10.2307/2982487

Queensland Government (2017) Queensland spatial catalogue. Department of natural resource and mines. http:// qldspatial.information.qld.gov.au/catalogue/custom/ index.page. Accessed 2nd June 2016.

R Core Team (2018) R: A language and environment for statistical computing. R Foundation for Statistical Computing, Vienna, Austria. http://www.R-project.org/

Ramos MM, Mohammed H, Zielinski-Gutierrez E, Hayden MH, Lopez JLR, Fournier M, Waterman SH (2008) Epidemic dengue and dengue hemorrhagic fever at the Texas-Mexico border: results of a household-based seroepidemiologic survey, December 2005. Am J Trop Med Hyg 78:364-369. https://doi.org/10.4269/ajtmh.2008.78.364

Reiter P, Amador MA, Anderson RA, Clark GG (1995) Short report: dispersal of Aedes aegypti in an urban area after blood feeding as demonstrated by rubidium-marked eggs. Am J Trop Med Hyg 52:177-179

Reiter P et al (2003) Texas lifestyle limits transmission of dengue virus. Emerg Infect Dis 9:86-89. https://doi.org/10. 3201/eid0901.020220

Russell RC, Currie BJ, Lindsay MD, Mackenzie JS, Ritchie SA, Whelan PI (2009) Dengue and climate change in Australia: predictions for the future should incorporate knowledge from the past. Med J Aust 190:265-268

Russell RC, Webb C, Williams C, Ritchie S (2005) Mark-release-recapture study to measure dispersal of the mosquito Aedes aegypti in Cairns. Queensland Aust Med Vet Entomol 19:451-457

State of Queensland (2015) Queensland dengue management plan 2015-2020. Queensland Health, 15 Butterfield St, Herston Qld 4006, Fortitude Valley BC 4006.

Taylor PD, Fahrig L, Henein K, Merriam G (1993) Connectivity is a vital element of landscape structure. Oikos 68:571-573. https://doi.org/10.2307/3544927

Trewin BJ (2018) Assessing the risk of establishment by the dengue vector, Aedes aegypti (L.)(Diptera: Culicidae), through rainwater tanks in Queensland: back to the future. University of Queensland.

Trewin BJ, Darbro JM, Jansen CC, Schellhorn NA, Zalucki MP, Hurst TP, Devine GJ (2017) The elimination of the dengue vector, Aedes aegypti, from Brisbane, Australia: the role of surveillance, larval habitat removal and policy. PLoS Negl 
Trop Dis 11:e0005848. https://doi.org/10.1371/journal. pntd.0005848

Trewin BJ, Darbro JM, Zalucki MP, Jansen CC, Schellhorn NA, Devine GJ (2019) Life on the margin: rainwater tanks facilitate overwintering of the dengue vector, Aedes aegypti, in a sub-tropical climate. PLOS ONE 14(4):e0211167

Trewin BJ, Kay BH, Darbro JM, Hurst TP (2013) Increased container-breeding mosquito risk owing to drought-induced changes in water harvesting and storage in Brisbane. Aust Int Health 5:251-258. https://doi.org/10.1093/ inthealth/iht 023

Trewin BJ, Pagendam DE, Zalucki MP, Darbro JM, Devine GJ, Jansen CC, Schellhorn NA (2020) Urban landscape features influence the movement and distribution of the Australian container-inhabiting mosquito vectors Aedes aegypti (Diptera: Culicidae) and Aedes notoscriptus
(Diptera: Culicidae). J Med Entomol 57:443-453. https:// doi.org/10.1093/jme/tjz187

Tun-Lin W, Kay B, Barnes A (1995) Understanding productivity, a key to Aedes aegypti surveillance. Am J Trop Med Hyg 53:595-601

Wolfinsohn M, Galun E (1953) A Method for determining the flight range of Aedes aegypti (Linn.). Bull Res Counc Isr 2:433-436

Zalucki M, Parry H, Zalucki J (2015) Movement and egg laying in monarchs: to move or not to move, that is the equation. Austral Ecol 41(2):154-167

Publisher's Note Springer Nature remains neutral with regard to jurisdictional claims in published maps and institutional affiliations. 